Brief Report

\title{
Role of JAK-STAT Pathway in Broiler Chicks Fed with Chestnut Tannins
}

\author{
Annah Lee ${ }^{1}$, Gabriela Cardoso Dal Pont ${ }^{1}$ (D), Michele Battaglia ${ }^{2}$, Ryan J. Arsenault ${ }^{3}$ and Michael H. Kogut ${ }^{4, *(D)}$ \\ 1 Department of Poultry Science, Texas A\&M University, College Station, TX 77843, USA; \\ annahlee89@tamu.edu (A.L.); gabrieladp@tamu.edu (G.C.D.P.) \\ 2 Silvateam s.p.a., San Michele Mondovi CN, 12080 Piedmont, Italy; michele@silvateam.com \\ 3 Department of Animal \& Food Sciences, University of Delaware, Newark, DE 19716, USA; rja@udel.edu \\ 4 Southern Plains Agricultural Research Center, USDA-ARS, College Station, TX 77845, USA \\ * Correspondence: mike.kogut@usda.gov
}

Citation: Lee, A.; Cardoso Dal Pont, G.; Battaglia, M.; Arsenault, R.J; Kogut, M.H Role of JAK-STAT Pathway in Broiler Chicks Fed with Chestnut Tannins. Animals 2021, 11, 337. https://doi.org/10.3390/ ani11020337

Academic Editor: Gunther Antonissen and Evy Goossens Received: 21 December 2020

Accepted: 25 January 2021

Published: 29 January 2021

Publisher's Note: MDPI stays neutral with regard to jurisdictional claims in published maps and institutional affiliations.

Copyright: (c) 2021 by the authors. Licensee MDPI, Basel, Switzerland. This article is an open access article distributed under the terms and conditions of the Creative Commons Attribution (CC BY) license (https:// creativecommons.org/licenses/by/ $4.0 /)$.
Simple Summary: Current bans on the use of antibiotics in livestock feed have led to increasing demand for alternatives to antibiotics (ATA). One popular alternative is chestnut tannins (ChT). While there is growing evidence of the immune benefits of using phytobiotics, such as ChT, there is currently minimal information on the effect of tannins on the immune pathway of the host. A previous study discovered a large upregulation of IL- 6 in broiler chicks at day 6 when provided with 1\% ChT from hatch. In regard to potential immune modulation, ChT appear to influence host immunity via an IL-6 mediated response, which could be beneficial in host defenses against pathogens at the early stages of broiler growth and development. A critical pathway identified in the regulation of the immune system is the JAK-STAT signaling pathway. The role of JAK-STAT pathway is altered by the addition of $\mathrm{ChT}$ in the diet. By demonstrating the changes in the kinome of the broiler model, the information in this study will provide further insights into potential ATA to improve poultry health.

Abstract: The objective of this study was to identify the phosphorylation events associated with host immunity with the inclusion of chestnut tannins (ChT) in the diet. A total of 200 male day-of-hatch Cobb 500 chicks were randomly assigned to two treatment groups, totaling 50 chicks per pen per experiment (this study was repeated two times). The treatments were as follows: (1) control feednormal starter feed $(n=50)$, and (2) $1 \%$ ChT inclusion feed $(n=50)$. The ceca were collected on each necropsy day for analysis via (1) a peptide array to provide tissue immunometabolism information from the host, and (2) quantitative PCR for mRNA expression. Of the top three immune pathways, the data identified the T-cell receptor signaling pathway, the chemokine signaling pathway, and the JAK-STAT signaling pathway. The results showed significantly altered phosphorylation of JAK and STAT peptides within the JAK-STAT pathway. These results support the mRNA expression data with the upregulated IL-6 response, due to the significant phosphorylation of IL6ST, JAK, and STAT peptides. In regard to immune modulation, ChT appear to influence host immunity via an IL-6 mediated response which could be beneficial in host defenses against pathogens at the early stages of broiler growth and development. Therefore, it is suggested that the role of the JAK-STAT pathway is altered by including ChT in the diet.

Keywords: tannins; JAK-STAT; kinome; immunity

\section{Introduction}

Antibiotic growth promoters (AGPs) were originally incorporated into livestock feed to promote better health and performance. However, with the ban of antibiotics in feed [1], there is a need for alternatives that will stimulate similar outcomes of AGPs. Recently, there has been increased interest in utilizing plant-based compounds, or phytobiotics, as antibiotic growth promoter alternatives, including chestnut tannins (ChT-Castanea sativa) [2,3]. Phytogenic compounds are viable candidates to replace AGPs due to their 
bioactive properties that emulate similar properties of antibiotics without causing antimicrobial resistance [4]. Plant-based tannins can be categorized into two major groups: condensed tannins and hydrolyzable tannins [5]. Tannins can be found in many plant species, mostly in the inedible portions of the plant such as the bark or wood [6,7]. This popular alternative to antibiotics (ATA) is already widely utilized on commercial farms for its overall health benefit and improved performance, although the mechanism of how it works is still not fully understood. Host gut immunity is better protected from pathogens via increased villus height:crypt depth ratio, improved intestinal mucosa to prevent tissue damage, production of pro-inflammatory and anti-inflammatory cytokines, and enhanced expression of tight junction proteins to modulate the immune system $[4,8-10]$.

Studying phosphorylation events provides information on the mechanism of posttranslational modification, which offers insight in cellular and tissue phenotypes [11,12]. The species-specific kinome array utilized in this study can measure the kinase activity on the tissue level, thereby allowing the observation of post-translational modifications and providing further insight into metabolic and immune function [13]. Our previous manuscript analyzed the metabolic phosphorylation events when ChT were included in the diet [14]. Therefore, the objective of this study was to analyze the outcome of specific immune phosphorylation events when broiler chicks were fed a ChT inclusion diet posthatch for up to 10 days. We analyzed multiple timepoints of chicken-specific kinomic immune changes in avian cecal tissue of chicks fed $1 \%$ ChT. Using this technique, we were able to identify specific phosphorylation-based immune post-translational signaling changes.

\section{Materials and Methods}

\subsection{Experimental Animals, Housing, and Treatments}

All experiments conducted were in accordance with guidelines set by the United States Department of Agriculture Animal Care and Use Committee (USDA ACUC \#2019001), which meets all federal requirements as defined in the Animal Welfare Act, and the Humane Care and Use of Laboratory Animals. A total of 200 male day-of-hatch Cobb 500 chicks were obtained from a local commercial hatchery and assigned to two treatment groups, totaling 50 chicks per pen per experiment. The treatments were as follows: (1) control feed-corn/soy starter feed $(n=50)$ and $(2) 1 \%$ ChT inclusion feed $(n=50)$. The chicks were randomly distributed into each group with fresh pine shavings, water, and the starter diet ad libitum. The chicks were kept under $96 \mathrm{~h}$ light and then transitioned to $18 \mathrm{~h}$ light and $6 \mathrm{~h}$ dark cycle until the end of the study. All treatments included a corn/soybean-based crumble diet, but differed in AGP or tannins inclusion. The diets were formulated to meet or exceed broiler requirements, as shown in Figure S1. The hydrolyzable ChT additive (Silvateam s.p.a. ( , Buenos Aires, Argentina) contained 75\% tannin content, supplemented with $94 \%$ dry matter, lignin and sugars. Each experimental process lasted 10 days. This study was repeated a total of two separate times.

\subsection{Sample Collection and Processing}

On each necropsy day, ten birds per group were selected by random, euthanized via cervical dislocation, and necropsied on days 2, 4, 6, 8, and 10 of each separate experiment. Both ceca were removed, flushed with phosphate buffered saline (PBS), and flash frozen in liquid nitrogen to preserve the kinase enzymatic activity. The frozen tissues were stored at $-80^{\circ} \mathrm{C}$ until further processing. Therefore, 100 ceca were collected on each separate experimental trial, for a total of 200 ceca collected at the end of both experiments.

\subsection{Bacterial Detection}

During each necropsy, the cecal contents $(0.25 \mathrm{~g} / \mathrm{bird})$ were collected and serially diluted to 1:100, 1:1000, 1:10,000, and 1:100,000 of $1 \times$ PBS. These contents were spread onto XLT4 for Salmonella detection for confirmation purposes. These plates were incubated at $37^{\circ} \mathrm{C}$ for $24 \mathrm{~h}$. Furthermore, an enrichment step was also performed for detection purposes: 
$100 \mathrm{uL}$ of the cecal contents were pre-enriched in Rappaport-Vassiliadis broth for $24 \mathrm{~h}$ at $37^{\circ} \mathrm{C}$. None of the birds showed the presence of Salmonella enterica throughout the study.

\subsection{Chicken-Specific Kinome (Peptide) Array}

For the phenotype readout, a peptide array was utilized to provide tissue immunometabolism information from the host. At three of the timepoints (days 4, 6, and 10), three whole ceca from three randomly selected birds-stored at $-80^{\circ} \mathrm{C}$ - were defrosted for analysis. Each distal end of the cecal sample was weighed to obtain a consistent $40 \mathrm{mg}$ sample for the array. The samples were homogenized using an Omni International Bead Ruptor Elite (Kennesaw, GA, USA) in $100 \mathrm{uL}$ of lysis buffer $(20 \mathrm{mM}$ Tris- $\mathrm{HCl} \mathrm{pH} 7.5,150 \mathrm{mM}$ $\mathrm{NaCl}, 1 \mathrm{mM}$ EDTA, $1 \mathrm{mM}$ ethylene glycol tetraacetic acid (EGTA), 1\% Triton X-100, $2.5 \mathrm{mM}$ sodium pyrophosphate, $1 \mathrm{mM}$ Na3VO4, $1 \mathrm{mM} \mathrm{NaF}, 1 \mu \mathrm{g} / \mathrm{mL}$ leupeptin, $1 \mathrm{~g} / \mathrm{mL}$ aprotinin, and $1 \mathrm{mM}$ phenylmethylsulphonyl fluoride). All products were obtained from Sigma Aldrich (St. Louis, MO, USA), unless indicated. Following homogenization, the peptide array protocol was carried out with previously described alterations $[13,15]$. The resulting tissue lysates were applied onto the PepStar peptide microarrays customized by JPT Peptide Technologies GmbH (Berlin, Germany).

\subsection{Real-Time Quantitative RT-PCR Assay}

Total RNA was extracted using a Qiagen RNeasy ${ }^{\circledR}$ Plus kit (Germantown, MD, USA) and evaluated with a NanoDrop ${ }^{\mathrm{TM}} 2000$ Spectrophotometer (Thermo Fisher Scientific, Waltham, MA, USA). Ceca stored in RNALater were used for RNA isolation with the Qiagen Rneasy Plus Kit. The ceca were cut longitudinally to expose the lumen, and any remaining fecal matter was gently removed with forceps. For each group, ten ceca were processed per experiment for qRT-PCR. Cytokine mRNA expression levels were ascertained using RT-PCR TaqMan based assay with $28 \mathrm{~S}$ as the reference gene. The RNAs were stored at $-80{ }^{\circ} \mathrm{C}$ until plate setup. The cytokines IL-1 $\beta$, IL-6, IL-8, IL-17, and IFN- $\gamma$ were quantified utilizing a previously published method [16]. The primer and probe sequences for amplification have been previously published and described $[17,18]$. The plates were run in the Applied Biosystems ABI StepOne Plus PCR system (ThermoFisher Scientific, Waltham, MA, USA) with the previously stated TaqMan Assay under the following conditions: one cycle of $48^{\circ} \mathrm{C}$ for $30 \mathrm{~min}, 95^{\circ} \mathrm{C}$ for $20 \mathrm{~s}$, and 40 cycles of $95^{\circ} \mathrm{C}$ for $3 \mathrm{~s}$ and $60^{\circ} \mathrm{C}$ for $30 \mathrm{~s}$. Results were calculated with the corrected 40- $C_{t}$ method [16] and expressed in fold change values. Each sample was run in triplicate for technical replication.

\subsection{Data Analysis: Kinome Array}

Once the peptide microarrays finished running $(n=27$ total for the three tested time points), data normalization was performed for the kinome array using the PIIKA2 online platform (http:/ / saphire.usask.ca/saphire/piika/index.html), a tool designed for in silico analysis of phosphorylation sites $[19,20]$. The array data were analyzed by conducting variance stabilization normalization and then performing a t-test, clustering, and pathway analysis for statistical data. Gene ontology (GO) and Kyoto Encyclopedia of Genes and Genomes (KEGG) pathway analyses were performed by uploading the statistically significant peptide lists to the Search Tool for the Retrieval of Interacting Genes (STRING) [21]. The protein-protein interaction networks were filtered by the minimum required interaction score with high confidence (0.700), in which the thickness of the connection lines indicate the strength of the data support.

\section{Results}

Broiler chicks fed $1 \%$ ChT had upregulated proinflammatory cytokine, specifically IL-6, mRNA expression in the ceca. Figure 1A,B depict each experimental fold change value across different days from the mRNA expression assay. The other tested pro-inflammatory cytokine results (IL-1 $\beta$, IL-8, IL-17, IFN- $\gamma$ ) can be found in Figure S3A-C. Of the proinflammatory cytokines tested, only IL-6 was found to be statistically significant in fold change 
differences (more than 32-fold) on days 6 and 10 in birds fed 1\% ChT compared to the controls. On day 4 , none of the cytokines tested was considered significant in fold change. By day 10, IL-6 still had a statistically significant three-fold change compared to the control, but not as elevated as it was on day 6 .

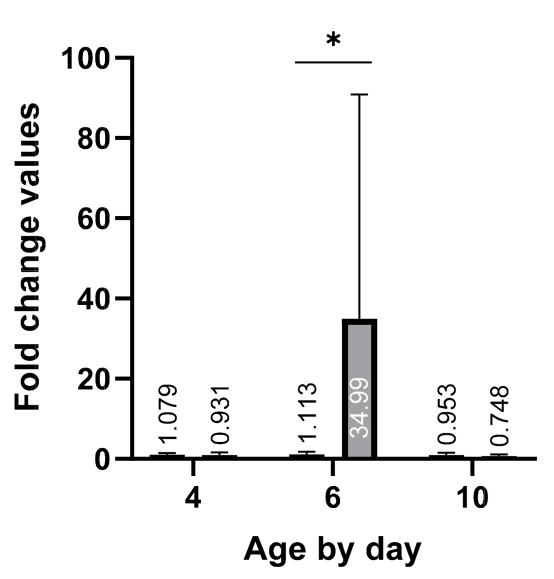

(A)

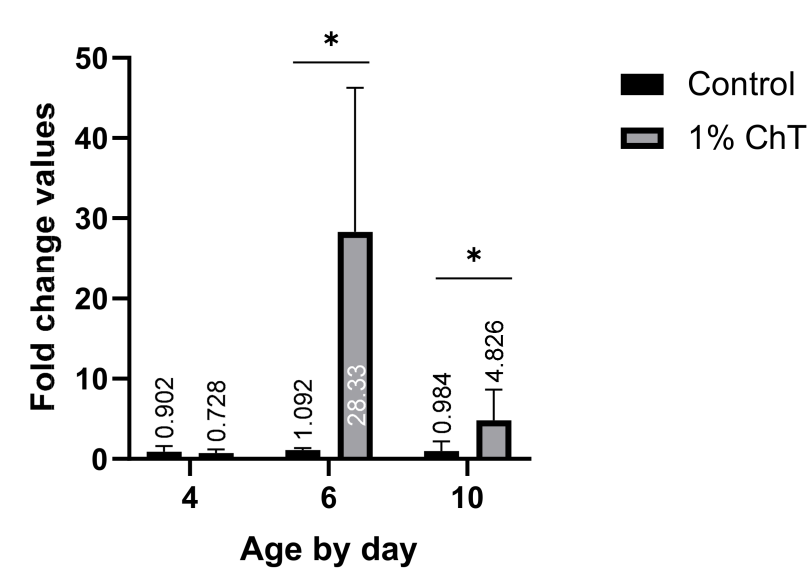

(B)

Figure 1. (A,B) IL-6 mRNA expression of cecal tissue from experimental chicks fed 1\% ChT inclusion diet, determined by quantitative RT-PCR: (A) First experimental fold changes by age tested comparison of averaged 1\% ChT inclusion group with control group; (B) Second experimental fold changes by age tested comparison of averaged 1\% ChT inclusion group with control group. ${ }^{*}=$ significantly different from the controls $(p \leq 0.05)$.

For the kinome array, one of the major phosphorylated pathways identified to be affected by ChT was the JAK-STAT signaling pathway for all days tested. Figure S2A-C display the complete interaction network of unique proteins from the kinome array for days 4,6 , and 10 . The clustered peptides indicate closely related connections. These figures also reveal the number of links between immune pathways (those without links are not confidently linked as interactions according to STRING-db). For each day tested, the JAKSTAT pathway was listed as one of the most altered immune pathways, as highlighted in Table 1). To further evaluate the phosphorylation events, the peptides involved directly or indirectly in the JAK-STAT pathways are listed in Table 2. JAK1 was significantly altered $(p \leq 0.01)$ on day 6 with no change in phosphorylation state on days 4 and 10 (as indicated by the hyphen). JAK2 $(p \leq 0.01)$ also appeared to be a target for altered phosphorylation, as seen on days 4 and 6 in 1\% ChT-fed chicks compared to the control. Another peptide of interest is the IL6ST peptide (Interleukin-6 receptor subunit beta), which had altered phosphorylation on days 4 and $10(p<0.05)$. The array also identified significant altered phosphorylation of STAT1, 3, and 5B activities on all tested days $(p<0.05)$. Therefore, the increased presence of JAK and STAT peptides indicated a stimulation in increased IL6 to stimulate JAK-STAT signaling pathway due to the inclusion of $1 \% \mathrm{ChT}$ in the diet.

The other affected immune pathways identified on the kinome array were T-cell receptor signaling pathway and chemokine signaling pathway. The resulting phosphorylation events within these pathways are listed in Tables 3 and 4. For the chemokine signaling pathway, CCR2 is one of the starting peptides of the pathway. This peptide was only significantly increased in phosphorylation on day 4, but no changes seen on days 6 and 10. Overall, neither the T-cell receptor nor the chemokine pathway showed significant activation by providing $1 \%$ ChT to broilers, as shown in Tables 3 and 4 . 
Table 1. Summarized table of Kyoto Encyclopedia of Genes and Genomes (KEGG) immune pathways at days 4, 6, and 10 comparing $1 \%$ ChT-fed birds and control birds. The hyphens indicate fewer than ten peptides and a lack of significance for our analysis. The JAK-STAT signaling pathway has been highlighted.

\begin{tabular}{|c|c|c|c|c|c|c|}
\hline \multicolumn{7}{|l|}{ Immune Pathways } \\
\hline & Day 4 & & Day 6 & & Day 10 & \\
\hline Identified Pathways & $\begin{array}{c}\text { Number } \\
\text { of } \\
\text { Peptides }\end{array}$ & $p$-Value & $\begin{array}{c}\text { Number } \\
\text { of } \\
\text { Peptides }\end{array}$ & $p$-Value & $\begin{array}{l}\text { Number } \\
\text { of Pep- } \\
\text { tides }\end{array}$ & $p$-Value \\
\hline Chemokine signaling pathway & 23 & $9.19 \times 10^{-18}$ & 26 & $9.19 \times 10^{-19}$ & 18 & $7.10 \times 10^{-13}$ \\
\hline $\mathrm{T}$ cell receptor signaling pathway & 14 & $7.55 \times 10^{-12}$ & 25 & $5.85 \times 10^{-23}$ & 12 & $7.50 \times 10^{-10}$ \\
\hline Jak-STAT signaling pathway & 13 & $1.38 \times 10^{-8}$ & 19 & $7.82 \times 10^{-13}$ & 15 & $1.31 \times 10^{-10}$ \\
\hline B cell receptor signaling pathway & 13 & $2.94 \times 10^{-12}$ & 19 & $3.27 \times 10^{-18}$ & 12 & $3.13 \times 10^{-11}$ \\
\hline Fc gamma R-mediated phagocytosis & 13 & $2.81 \times 10^{-11}$ & 13 & $4.06 \times 10^{-10}$ & - & - \\
\hline TNF signaling pathway & 13 & $2.21 \times 10^{-10}$ & 21 & $1.17 \times 10^{-17}$ & 10 & $1.72 \times 10^{-7}$ \\
\hline Wnt signaling pathway & 13 & $4.24 \times 10^{-9}$ & 11 & $3.30 \times 10^{-6}$ & - & - \\
\hline Apoptosis & 12 & $2.09 \times 10^{-8}$ & 19 & $5.79 \times 10^{-14}$ & - & - \\
\hline Toll-like receptor signaling pathway & 11 & $1.46 \times 10^{-8}$ & 20 & $6.03 \times 10^{-17}$ & 13 & $9.43 \times 10^{-11}$ \\
\hline Natural killer cell mediated cytotoxicity & 11 & $8.57 \times 10^{-8}$ & 16 & $1.76 \times 10^{-11}$ & - & - \\
\hline NOD-like receptor signaling pathway & 10 & $8.08 \times 10^{-6}$ & 18 & $1.22 \times 10^{-11}$ & 12 & $1.11 \times 10^{-7}$ \\
\hline Th17 cell differentiation & 10 & $1.43 \times 10^{-7}$ & 22 & $3.36 \times 10^{-19}$ & 10 & $1.11 \times 10^{-7}$ \\
\hline IL-17 signaling pathway & - & - & 16 & $3.62 \times 10^{-13}$ & - & - \\
\hline $\begin{array}{c}\text { Inflammatory mediator regulation of } \\
\text { TRP channels }\end{array}$ & - & - & 10 & $6.08 \times 10^{-7}$ & - & - \\
\hline
\end{tabular}

Table 2. Proteins from the JAK-STAT signaling pathway that displayed a statistically significant change in phosphorylation. The UniProt ID helps identify the protein. Positive fold change values indicate phosphorylation events; negative values indicate dephosphorylation events. Only proteins that displayed a $p$-value of less than 0.05 are shown.

\begin{tabular}{|c|c|c|c|c|c|c|c|c|}
\hline \multirow[b]{4}{*}{ Proteins } & \multirow[b]{4}{*}{ UniProt ID } & \multirow[b]{4}{*}{ p-Site } & \multicolumn{6}{|c|}{ JAK-STAT Signaling Pathway } \\
\hline & & & \multicolumn{6}{|c|}{ Days of Necropsy } \\
\hline & & & \multicolumn{2}{|l|}{4} & \multicolumn{2}{|l|}{6} & \multicolumn{2}{|l|}{10} \\
\hline & & & Fold Change & $p$-Value & Fold Change & $p$-Value & Fold Change & $p$-Value \\
\hline AKT3 & Q9Y243 & S476/T305 & -1.046 & $<0.01$ & -1.041 & 0.026 & -1.023 & 0.034 \\
\hline CCND1 & P24385 & T286 & - & - & 1.071 & 0.015 & - & - \\
\hline EGFR & P00533 & Y1069 & - & - & 1.032 & 0.041 & - & - \\
\hline EP300 & Q09472 & S89 & - & - & - & - & 1.052 & 0.031 \\
\hline GRB2 & P62993 & Y209 & - & - & -1.063 & 0.02 & 1.044 & 0.017 \\
\hline IL12B & P29460 & Y314 & - & - & -1.048 & 0.03 & - & - \\
\hline IL6ST & P40189 & Y915/Y759 & 1.058 & 0.026 & -1.039 & 0.03 & 1.041 & 0.039 \\
\hline JAK1 & P23458 & Y1034 & - & - & 1.098 & $<0.01$ & - & - \\
\hline JAK2 & O60674 & Y1007 & -1.059 & 0.027 & -1.042 & $<0.01$ & - & - \\
\hline MTOR & P42345 & S2481 & 1.061 & 0.042 & -1.058 & 0.01 & -1.041 & 0.036 \\
\hline PDGFRA & P16234 & Y720 & - & - & 1.127 & $<0.01$ & -1.064 & $<0.01$ \\
\hline PDGFRB & P09619 & Y579 & -1.115 & $<0.01$ & -1.061 & 0.019 & -1.061 & 0.046 \\
\hline PIK3CB & $\mathrm{P} 42338$ & Y425/S1070 & 1.086 & $<0.01$ & - & - & -1.049 & 0.022 \\
\hline PIK3R1 & P27986 & S608/Y467 & 1.058 & 0.028 & -1.025 & 0.045 & -1.076 & $<0.01$ \\
\hline PIK3R2 & O00459 & Y365 & 1.052 & 0.038 & -1.039 & 0.04 & - & - \\
\hline PIM1 & P11309 & S189 & - & - & -1.046 & $<0.01$ & -1.043 & 0.036 \\
\hline
\end{tabular}


Table 2. Cont.

\begin{tabular}{|c|c|c|c|c|c|c|c|c|}
\hline \multirow[b]{4}{*}{ Proteins } & \multirow[b]{4}{*}{ UniProt ID } & \multirow[b]{4}{*}{ p-Site } & \multicolumn{6}{|c|}{ JAK-STAT Signaling Pathway } \\
\hline & & & \multicolumn{6}{|c|}{ Days of Necropsy } \\
\hline & & & \multicolumn{2}{|l|}{4} & \multicolumn{2}{|l|}{6} & \multicolumn{2}{|l|}{10} \\
\hline & & & Fold Change & $p$-Value & Fold Change & $p$-Value & Fold Change & $p$-Value \\
\hline RAF1 & P04049 & S259 & 1.053 & 0.03 & -1.048 & 0.048 & - & - \\
\hline SOCS3 & O14543 & Y204 & -1.069 & $<0.01$ & -1.052 & $<0.01$ & - & - \\
\hline SOS1 & Q07889 & S1193 & - & - & - & - & 1.036 & 0.029 \\
\hline STAM2 & O75886 & Y371 & - & - & - & - & -1.057 & 0.017 \\
\hline STAT1 & P42224 & Y701 & 1.057 & 0.048 & 1.060 & 0.033 & 1.034 & 0.034 \\
\hline STAT3 & P40763 & Y705/S727 & 1.067 & 0.015 & 1.078 & 0.031 & 1.046 & 0.044 \\
\hline STAT5B & P51692 & Y699 & -1.071 & $<0.01$ & 1.061 & $<0.01$ & 1.087 & $<0.01$ \\
\hline
\end{tabular}

Table 3. Chemokine signaling pathway in chicken ceca on days 4,6 , and 10 of age. The results of this table indicate no clear path utilized by the host immunity of the chemokine signaling pathway other than presence of JAK2 and STAT proteins, which would suggest directionality toward the JAK-STAT pathway.

\begin{tabular}{|c|c|c|c|}
\hline \multicolumn{4}{|c|}{ Chemokine Signaling Pathway } \\
\hline Proteins & Day 4 & Day 6 & Day 10 \\
\hline AKT3 & $\downarrow$ & $\downarrow$ & $\downarrow$ \\
\hline ARRB1 & $\downarrow$ & $\downarrow$ & - \\
\hline ARRB2 & - & $\downarrow$ & - \\
\hline CHUK & - & $\uparrow$ & - \\
\hline CRK & - & - & $\uparrow$ \\
\hline CRKL & $\downarrow$ & - & - \\
\hline CRR2 & $\uparrow$ & - & - \\
\hline GRB2 & - & $\downarrow$ & $\uparrow$ \\
\hline GRK5 & $\downarrow$ & $\downarrow$ & - \\
\hline GSK3A & $\downarrow$ & $\uparrow$ & $\downarrow$ \\
\hline GSK3B & $\downarrow$ & $\uparrow$ & $\uparrow$ \\
\hline JAK2 & $\downarrow$ & $\downarrow$ & - \\
\hline LYN & - & $\uparrow$ & - \\
\hline MAP2K1 & $\uparrow$ & - & - \\
\hline NFKB1 & - & $\uparrow$ & - \\
\hline NFKBIA & - & $\uparrow$ & $\downarrow$ \\
\hline PAK1 & $\downarrow$ & - & - \\
\hline PIK3R1 & $\uparrow$ & $\downarrow$ & $\downarrow$ \\
\hline PIK3R2 & $\uparrow$ & $\downarrow$ & - \\
\hline PRKCD & $\downarrow$ & $\downarrow$ & - \\
\hline PTK2 & $\uparrow$ & $\uparrow$ & $\downarrow$ \\
\hline PTK2B & $\uparrow$ & $\uparrow$ & $\uparrow$ \\
\hline PXN & $\downarrow$ & $\uparrow$ & $\downarrow$ \\
\hline RAF1 & $\uparrow$ & $\downarrow$ & - \\
\hline SHC1 & - & $\uparrow$ & - \\
\hline SHC3 & - & $\uparrow$ & - \\
\hline SRC & - & $\downarrow$ & $\uparrow$ \\
\hline STAT1 & $\uparrow$ & $\uparrow$ & $\uparrow$ \\
\hline STAT3 & $\uparrow$ & $\uparrow$ & $\uparrow$ \\
\hline STAT5B & $\downarrow$ & $\uparrow$ & $\uparrow$ \\
\hline
\end{tabular}


Table 4. T-cell receptor signaling pathway in chicken ceca on days 4,6 , and 10 of age. The results of this table indicate no clear path utilized by the host immunity of the T-cell receptor signaling pathway.

\begin{tabular}{cccc}
\hline & T Cell Receptor Signaling Pathway & \\
\hline Proteins & Day 4 & Day $\mathbf{6}$ & Day 10 \\
\hline AKT3 & $\downarrow$ & $\downarrow$ & $\downarrow$ \\
CHUK & - & $\downarrow$ & - \\
FOS & - & $\uparrow$ & - \\
FYN & $\downarrow$ & $\downarrow$ & $\uparrow$ \\
GRB2 & - & $\downarrow$ & $\uparrow$ \\
GSK3B & $\downarrow$ & $\uparrow$ & - \\
JUN & $\downarrow$ & $\downarrow$ & - \\
LCK & - & $\uparrow$ & - \\
MAP2K1 & $\uparrow$ & - & $\uparrow$ \\
MAP2K2 & - & $\downarrow$ & $\uparrow$ \\
MAP3K7 & $\downarrow$ & $\uparrow$ & - \\
NFATC1 & $\uparrow$ & - & $\uparrow$ \\
NFATC2 & - & $\downarrow$ & - \\
NFATC3 & $\uparrow$ & $\downarrow$ & $\downarrow$ \\
NFKB1 & - & $\uparrow$ & - \\
NFKBIA & - & $\uparrow$ & - \\
PAK1/2 & $\downarrow$ & $\downarrow$ & $\downarrow$ \\
PDPK1 & $\downarrow$ & $\downarrow$ & $\downarrow$ \\
PIK3R1 & $\uparrow$ & $\downarrow$ & - \\
PIK3R2 & $\uparrow$ & $\downarrow$ & - \\
PTPRC & - & $\downarrow$ & $\downarrow$ \\
RAF1 & - & $\downarrow$ & $\downarrow$ \\
ZAP70 & increasing phosphorylated from noninfected control; & $\downarrow$ \\
$(p \leq 0.05)$ decreasing phosphorylated from noninfected control; - & not significant from noninfected control.
\end{tabular}

\section{Discussion}

Demands for alternatives to antibiotics, such as ChT, in livestock feed have increased globally in the past several years. Growing research is showing the importance of the gastrointestinal system and its role in host immune modulation [22-24]. Numerous reviews have already been published regarding the evidence of ChT in improving overall host health and immunity during pathogenic events, but relatively little is known about the functional role that ChT play in immunity in a non-infection model. One of the most altered peptide pathways from the present study is the JAK-STAT signaling pathway, which is crucial for growth, energy, immunity, and overall development of the animal. This pathway is also important for its connection to IL-6 regulation, which was heavily upregulated on day 6 of the mRNA expression array (Figure 1A,B). Figure S2A-C display the complete interaction network of unique proteins from the kinome array for days 4,6, and 10 . This network reveals the strong patterns towards immune connections, especially with the JAK-STAT signaling pathway (affiliated peptides indicated in the purple circles on each time point). Based on our findings, the kinomic analysis demonstrates the importance of a phytobiotic incorporation in feed, providing further evidence of its potential as a key ATA. These results may also provide useful information regarding how the JAK-STAT pathway is modified with the inclusion of phytobiotics in livestock diet.

The JAK family proteins are the initial receptors present to initiate the JAK-STAT pathway. In the chicken, JAK1 expression is found in the bursa and thymus at high levels, perhaps due to the post-translational modification activity of JAK1 kinase in these locations [25]. A previous study discovered interactions between avian JAK1 and STAT1/3/5B affected different cytokine families, including IL-6 and IL-10, in the gut during a disease state in the birds [26]. Another study in broiler chickens found upregulated JAK1 and STAT1,3,5B mRNA levels led to increased expressions of IL-2, IL-4, IL-6, IL-7, IL-10, IL-22, and IFN- $\alpha$ [26]. The mRNA expression data revealed an upregulation of statistically signif- 
icant fold change on days 6 and 10, while the other proinflammatory cytokines were not found to be statistically significant. This provides evidence of an IL-6 immune mediated proinflammatory response, as opposed to initiating T-cell receptors or chemokine receptors. Although Table 1 shows that the T-cell receptor signaling pathway and chemokine signaling pathway are the top two pathways with most altered peptides, this information only provides altered peptide numbers and not detailed phosphorylation or dephosphorylation events. The chemokine signaling pathway appeared to follow the CCR2-GNB network of signaling pathways; however, with the significantly increased phosphorylation in STAT1,3,5B peptides and significant phosphorylation presence of JAK2, this points to even greater evidence of the JAK-STAT pathway being activated from the chemokine signaling pathway. As reflected by our mRNA expression results, chemokine IL- 8 did not show any significant fold change between the $1 \% \mathrm{ChT}$ group and the control group. The T-cell receptor pathway showed less clear pathway information, even though it was the immune pathway with the most altered peptides. However, this pathway is an important precursor pathway that promotes signaling cascades for other immune pathways, including the PI3K-Akt signaling pathway, the MAPK signaling pathway, and the NF- $\beta$ signaling pathway [27]. As shown in Table 4, the direct peptides involved in the pathway do not indicate a clear T-cell function, which may be the reason why our IL-1 family cytokines did not show significant fold changes.

The kinome data provides further evidence of this trend with the decreasing phosphorylation events within the T-cell receptor signaling pathway and chemokine signaling pathway across all three days tested; see Tables 3 and 4 . The kinomic data for day 6 seem to follow the IL-6 family of cytokine signaling for the JAK-STAT pathway, shown in Figure S4: IL-6, which promotes the growth and differentiation of different cell types and can activate the pathway by phosphorylating JAK1,2 and then activating downstream signaling STAT family proteins, such as STAT1 and STAT3 [28,29]. Interestingly, the JAK-STAT signaling pathway was the only one that had IL6R out of the 13 signal pathways involved in avian embryonic stem cell differentiation [29]. A previous in vivo experiment suggested that IL-6 can regulate the JAK-STAT pathway by decreasing JAK2 and increasing levels of STAT3 [28,30], which is supported by our results. Our in vivo kinomic results, shown in Table 2, observed increasing phosphorylation of IL6ST on days 4 and 10, apart from dephosphorylation observed on day 6. This is especially noteworthy in the biological processes, in which phosphorylation of IL6ST at this site would alter signaling pathway regulation and transcription processes [31,32]. This dephosphorylation may be due to the host immune system attempting to regulate the strong pro-inflammatory response via the IL-6 mediated pathway. Notably, SOCS3, the primary inhibitor of IL-6 signaling, is present on days four and six in our data. The negative values as seen in this present study associated with the SOCS protein would indicate less presence of negative regulation of the JAK-STAT pathway [32-34]. This would potentially explain the negative values associated with the JAK2 and IL6ST on days four and six, respectively.

This study aimed to provide phosphorylation details regarding the broiler chick immune response when $\mathrm{ChT}$ are included into the grower diet. The ChT appear to be acting as an immune activator due to the high upregulation of IL-6 proinflammatory cytokine. In considering practical application, recent studies suggest the usage of encouraging proinflammatory states for beneficial purposes of stimulating the immune system at early stages to improve resistance against pathogens [23]. As a key signaling pathway involved in regulating innate immunity and adaptive immunity, the JAK-STAT pathway is crucial to study when considering using ATA such as ChT. Therefore, this study shows evidence of the modulatory effect of ChT on the host, suggesting a role of JAK-STAT pathway in altered immunomodulation via IL-6 signal transduction. Future experiments will investigate the usage of ChT as antimicrobial immunomodulators on the local cecal level. 


\section{Conclusions}

Supplementing broiler diets on day 6 with 1\% ChT provided evidence of modulation based on the alteration of JAK-STAT signaling pathway in the host. Based on the kinome array, the JAK-STAT pathway was one of the most altered signaling pathways involved in the immune response. This was further evidenced by the significant upregulation of IL-6 proinflammatory cytokine on day 6 . This suggests a role of the JAK-STAT pathway in altered immunomodulation via IL-6 signal transduction pathway. However, more research will need to be conducted to further elucidate the effects of ChT on immunity and its role.

Supplementary Materials: The following are available online at https:/ / www.mdpi.com/2076-2 615/11/2/337/s1, Figure S1: Calculated composition of corn/soy starter diets. The total basal diet contained 1,365 kcal/lb, Figure S2A-C: STRING-db network of interactions. Purple circles indicate the JAK-STAT peptides affiliated to the pathway; the other colors indicate regulatory pathways affecting the JAK-STAT pathway: (A) Protein-protein interactions between proteins included in the day 4 chicken immune peptide array and metabolic peptide array; (B) Protein-protein interactions between proteins included in the day 6 chicken immune peptide array and metabolic peptide array; (C) Protein-protein interactions between proteins included in the day 10 chicken immune peptide array and metabolic peptide array, Figure S3A-C. mRNA expression of cecal mRNA from experimental chicks fed 1\% ChT inclusion diet, determined by quantitative RT-PCR. (A) Day 4 fold changes by cytokines tested comparing averaged $1 \% \mathrm{ChT}$ inclusion group with control group; (B) Day 6 fold changes by cytokines tested comparing averaged 1\% ChT inclusion group with control group; (C) Day 10 fold changes by cytokines tested comparing averaged 1\% ChT inclusion group with control group. Data represent the median values from two separate experiments. ${ }^{*}=$ significantly different from the controls $(p \leq 0.05)$, Figure S4. The phosphorylation of STAT proteins activates the initiation of gene transcription. The black arrows indicate phosphorylation, and the red arrow indicates inhibitory action.

Author Contributions: A.L. conceived and designed the experiment. A.L., M.B. and M.H.K. supervised the project. A.L. and G.C.D.P. performed the necropsies and collected the experimental data. A.L., R.J.A. and M.H.K. interpreted the data. A.L. performed the data analysis. All authors contributed to reviewing the manuscript and gave final approval for the final publishable version. All authors have read and agreed to the published version of the manuscript.

Funding: This project was funded by the United States Department of Agriculture, Agricultural Research Service Project \#3091-32000-034-00.

Institutional Review Board Statement: The study was approved by the Animal Care and Use Committee of the United States Department of Agriculture (USDA ACUC \#2019001), which meets all federal requirements as defined in the Animal Welfare Act, and the Humane Care and Use of Laboratory Animals.

Data Availability Statement: No new data were created or analyzed in this study. Data sharing is not applicable to this article.

Acknowledgments: The authors thank Reiley Street, Kenneth Genovese, Haiqi He, Christina Swaggerty, Cristiano Bortoluzzi of the United States Department of Agriculture, Agriculture Research Service for their assistance throughout the duration of the project. The chestnut tannin extract was provided by Silvateam s.p.a., San Michele Mondovi, Italy.

Conflicts of Interest: The authors declare that the research was conducted in the absence of any commercial or financial relationships that could be construed as a potential conflict of interest.

Ethics Statement: All experiments conducted were in accordance with guidelines set by the United States Department of Agriculture Animal Care and Use Committee (USDA ACUC \#2019001), which meets all federal requirements as defined in the Animal Welfare Act, and the Human Care and Use of Laboratory Animals. 


\section{References}

1. Lillehoj, H.; Liu, Y.; Calsamiglia, S.; Fernandez-Miyakawa, M.E.; Chi, F.; Cravens, R.L.; Oh, S.; Gay, C.G. Phytochemicals as antibiotic alternatives to promote growth and enhance host health. Vet. Res. 2018, 49, 1. [CrossRef]

2. Ren, H.; Vahjen, W.; Dadi, T.; Saliu, E.-M.; Boroojeni, F.G.; Zentek, J. Synergistic effects of probiotics and phytobiotics on the intestinal microbiota in young broiler chicken. Microorganisms 2019, 7, 684. [CrossRef]

3. Diaz Carrasco, J.M.; Redondo, L.M.; Redondo, E.A.; Dominguez, J.E.; Chacana, A.P.; Fernandez Miyakawa, M.E. Use of plant extracts as an effective manner to control Clostridium perfringens induced necrotic enteritis in poultry. BioMed. Res. Int. 2016, 2016, 3278359. [CrossRef]

4. Díaz Carrasco, J.M.; Redondo, E.A.; Pin Viso, N.D.; Redondo, L.M.; Farber, M.D.; Fernández Miyakawa, M.E. Tannins and bacitracin differentially modulate gut microbiota of broiler chickens. BioMed. Res. Int. 2018, 2018, 1879168. [CrossRef]

5. Huang, Q.; Liu, X.; Zhao, G.; Hu, T.; Wang, Y. Potential and challenges of tannins as an alternative to in-feed antibiotics for farm animal production. Anim. Nutr. 2018, 4, 137-150. [CrossRef]

6. Brus, M.; Gradišnik, L.; Trapečar, M.; Škorjanc, D.; Frangež, R. Beneficial effects of water-soluble chestnut (Castanea sativa mill.) tannin extract on chicken small intestinal epithelial cell culture. Poult. Sci. 2018, 97, 1271-1282. [CrossRef] [PubMed]

7. Molino, S.; Casanova, N.A.; Rufián Henares, J.Á.; Fernandez Miyakawa, M.E. Natural tannin wood extracts as a potential food ingredient in the food industry. J. Agric. Food Chem. 2020, 68, 2836-2848. [CrossRef]

8. Liu, H.W.; Li, K.; Zhao, J.S.; Deng, W. Effects of chestnut tannins on intestinal morphology, barrier function, pro-inflammatory cytokine expression, microflora and antioxidant capacity in heat-stressed broilers. J. Anim. Physiol. Anim. Nutr. 2018, 102, 717-726. [CrossRef]

9. Song, P.; Zhang, R.; Wang, X.; He, P.; Tan, L.; Ma, X. Dietary grape-seed procyanidins decreased postweaning diarrhea by modulating intestinal permeability and suppressing oxidative stress in rats. J. Agric. Food Chem. 2011, 59, 6227-6232. [CrossRef]

10. Williams, A.R.; Andersen-Civil, A.I.S.; Zhu, L.; Blanchard, A. Dietary phytonutrients and animal health: Regulation of immune function during gastrointestinal infections. J. Anim. Sci. 2020, 98, 1-11. [CrossRef]

11. Manning, G. The protein kinase complement of the human genome. Science 2002, 298, 1912-1934. [CrossRef] [PubMed]

12. Arsenault, R.; Griebel, P.; Napper, S. Peptide arrays for kinome analysis: New opportunities and remaining challenges. Proteomics 2011, 11, 4595-4609. [CrossRef] [PubMed]

13. Jalal, S.; Arsenault, R.; Potter, A.A.; Babiuk, L.A.; Griebel, P.J.; Napper, S. Genome to kinome: Species-specific peptide arrays for kinome analysis. Sci. Signal. 2009, 2, pl1. [CrossRef] [PubMed]

14. Lee, A.; Cardoso Dal Pont, G.; Farnell, M.B.; Jarvis, S.; Battaglia, M.; Arsenault, R.J.; Kogut, M.H. Supplementing chestnut tannins in the broiler diet mediates a metabolic phenotype of the ceca. Poult. Sci. 2020, 100, 47-54. [CrossRef]

15. Arsenault, R.J.; Lee, J.T.; Latham, R.; Carter, B.; Kogut, M.H. Changes in immune and metabolic gut response in broilers fed $\beta$-mannanase in $\beta$-mannan-containing diets. Poult. Sci. 2017, 96, 4307-4316. [CrossRef]

16. Eldaghayes, I.; Rothwell, L.; Williams, A.; Withers, D.; Balu, S.; Davison, F.; Kaiser, P. Infectious bursal disease virus: Strains that differ in virulence differentially modulate the innate immune response to infection in the chicken bursa. Viral Immunol. 2006, 19, 83-91. [CrossRef]

17. Kogut, M.H.; Rothwell, L.; Kaiser, P. Differential regulation of cytokine gene expression by avian heterophils during receptormediated phagocytosis of opsonized and nonopsonized Salmonella enteritidis. J. Interferon Cytokine Res. 2003, 23, $319-327$. [CrossRef]

18. Kaiser, P.; Wigley, P.; Burnside, J.; Barrow, P.A.; Galyov, E.E.; Rothwell, L. Differential cytokine expression in avian cells in response to invasion by Salmonella typhimurium, Salmonella enteritidis and Salmonella gallinarum. Microbiology 2000, 146, 3217-3226. [CrossRef]

19. Trost, B.; Kindrachuk, J.; Määttänen, P.; Napper, S.; Kusalik, A. PIIKA 2: An expanded, web-based platform for analysis of kinome microarray data. PLoS ONE 2013, 8, e80837. [CrossRef]

20. Li, Y.; Arsenault, R.J.; Trost, B.; Slind, J.; Griebel, P.J.; Napper, S.; Kusalik, A. A systematic approach for analysis of peptide array kinome data. Sci. Signal. 2012, 5, pl2. [CrossRef]

21. Szklarczyk, D.; Morris, J.H.; Cook, H.; Kuhn, M.; Wyder, S.; Simonovic, M.; Santos, A.; Doncheva, N.T.; Roth, A.; Bork, P.; et al. The STRING database in 2017: Quality-controlled protein-protein association networks. Nucleic Acids Res. 2017, 45, D362-D368. [CrossRef] [PubMed]

22. Kogut, M.H.; Yin, X.; Yuan, J.; Broom, L. Gut health in poultry. CAB Rev. 2017, 12, 1-7. [CrossRef]

23. Broom, L.J.; Kogut, M.H. Deciphering desirable immune responses from disease models with resistant and susceptible chickens. Poult. Sci. 2019, 98, 1634-1642. [CrossRef] [PubMed]

24. Kogut, M.H. The effect of microbiome modulation on the intestinal health of poultry. Anim. Feed Sci. Tech. 2019, 250, 32-40. [CrossRef]

25. Bartunek, P.; Koritschoner, N.P.; Brett, D.; Zenke, M. Molecular cloning, expression and evolutionary analysis of the avian tyrosine kinase JAK1. Gene 1999, 230, 129-136. [CrossRef]

26. Truong, A.D.; Rengaraj, D.; Hong, Y.; Hoang, C.T.; Hong, Y.H.; Lillehoj, H.S. Analysis of JAK-STAT signaling pathway genes and their microRNAs in the intestinal mucosa of genetically disparate chicken lines induced with necrotic enteritis. Vet. Immunol. Immunopathol. 2017, 187, 1-9. [CrossRef] 
27. Huang, Y.; Wange, R.L. T cell receptor signaling: Beyond complex complexes. J. Biol. Chem. 2004, 279, 28827-28830. [CrossRef]

28. Morris, R.; Kershaw, N.J.; Babon, J.J. The molecular details of cytokine signaling via the JAK/STAT pathway. Protein Sci. 2018, 27, 1984-2009.

29. Zhang, Y.; Zhang, L.; Zuo, Q.; Wang, Y.; Zhang, Y.; Xu, Q.; Li, B.; Chen, G. JAK-STAT signaling regulation of chicken embryonic stem cell differentiation into male germ cells. In Vitro Cell. Dev. Biol. 2017, 53, 728-743. [CrossRef]

30. Cousins, E.; Gao, Y.; Sandford, G.; Nicholas, J. Human herpesvirus 8 viral interleukin-6 signaling through gp130 promotes virus replication in primary effusion lymphoma and endothelial cells. J. Virol. 2014, 88, 12167-12172.

31. Stross, C.; Radtke, S.; Clahsen, T.; Gerlach, C.; Volkmer-Engert, R.; Schaper, F.; Heinrich, P.C.; Hermanns, H.M. Oncostatin M receptor-mediated signal transduction is negatively regulated by SOCS3 through a receptor tyrosine-independent mechanism. $J$. Biol. Chem. 2006, 281, 8458-8468. [CrossRef] [PubMed]

32. Rawlings, J.S. The JAK/STAT signaling pathway. J. Cell Sci. 2004, 117, 1281-1283. [CrossRef] [PubMed]

33. Seif, F.; Khoshmirsafa, M.; Aazami, H.; Mohsenzadegan, M.; Sedighi, G.; Bahar, M. The role of JAK-STAT signaling pathway and its regulators in the fate of T helper cells. Cell Commun. Signal. 2017, 15, 23. [CrossRef] [PubMed]

34. Tanaka, T.; Narazaki, M.; Kishimoto, T. IL-6 in inflammation, immunity, and disease. Cold Spring Harb. Perspect. Biol. 2014, 6, a016295. [CrossRef] [PubMed] 